\title{
On solution of an optimal control problem governed by a linear wave equation
}

Yesim Sarac

Department of Mathematics, Faculty of Science, Ataturk University, Erzurum, Turkey

Received: 20 August 2016, Accepted: 8 September 2016

Published online: 14 December 2016.

\begin{abstract}
This paper studies the minimization problem governed by a wave equation with homogeneous Neumann boundary condition and where the control function is a initial velocity of the system. We give necessary conditions for the existence and uniqueness of the optimal solution. We get the Frechet derivation of the cost functional via the solution of the corresponding adjoint problem. We construct a minimizing sequence and show that the limit of the minimizing sequence is the solution of the optimal control problem.
\end{abstract}

Keywords: Wave equation, adjoint problem, optimization, regularization.

\section{Introduction and problem formulation}

In this study, we consider an optimal control problem for a wave equation with homogeneous Neumann boundary conditions. We determine the unknown function $v(x)$ which is the initial velocity of the system in the closed and convex subset $U_{a d}$ of $L_{2}(0, l)$ from the target $u(0, t ; v)$, using $L_{2}-$ norm.

Choose a control $v(x)$ and a corresponding $u$ such that the pair $(v, u)$ minimizes the function

$$
J_{\alpha}(v)=\int_{0}^{T}[u(0, t ; v)-y(t)]^{2} d t+\alpha \int_{0}^{l} v^{2} d x
$$

subject to the linear hyperbolic problem;

$$
\begin{aligned}
& u_{t t}-a^{2} u_{x x}=F(x, t), \quad(x, t) \in \Omega:=(0, l) \times(0, T] \\
& u(x, 0)=\varphi(x), \quad u_{t}(x, 0)=v(x), \quad x \in(0, l) \\
& u_{x}(0, t)=0, \quad u_{x}(l, t)=0, \quad t \in(0, T]
\end{aligned}
$$

where $y$ is desired target function in $L_{2}(0, T)$ and $\varphi$ and $F$ are known functions satisfying the following conditions:

$$
\varphi(x) \in H^{1}(0, l), v(x) \in L_{2}(0, l), \quad F(x, t) \in L_{2}(\Omega)
$$

With the choice of the functional in(1), we mentioned the observation of $u(0, t ; v)$ in $L_{2}(0, T)$ for the control $v(x) \in L_{2}(0, l)$.

The aim of this work is to obtain suitable function $v_{*}$ which approaches the solution of the problem (2) at the left boundary $x=0$ to desired target $y(t) \in L_{2}(0, T)$. Another word, we want to determine the optimal function $v_{*}$ in a 
admissible set $U_{a d}$ such that

$$
J_{\alpha}\left(v_{*}\right) \leq J_{\alpha}(v), \forall v \in U_{a d}
$$

Here $\alpha>0$ is a regularization parameter which ensures both the uniqueness of the solution and a balance between the norms $\|u(0, t ; v)-y(t)\|_{L_{2}(0, T)}^{2}$ and $\|v\|_{L_{2}(0, l)}^{2}$. Detailed information as regards the regularization parameter can be found in [1]. The term $\|v\|_{L_{2}}^{2}$ is called penalization term; its role is to avoid using too large controls in the minimization of $J_{\alpha}(v)$.

The optimal control problems with different cost functionals for the hyperbolic systems have been studied by different authors $[2,6]$.

Kowalewski [7] considered the control $v$ in the hyperbolic problem

$$
\begin{aligned}
& \frac{\partial^{2} y}{\partial t^{2}}+A y+y(x, t-h)=u, \quad x \in \Omega, t \in(0, T) \\
& y\left(x, t^{\prime}\right)=\Phi_{0}\left(x, t^{\prime}\right), \quad x \in \Omega, t^{\prime} \in[-h, 0) \\
& y(x, 0)=0, y^{\prime}(x, 0)=v, \quad x \in \Omega \\
& y(x, t)=0, \quad x \in \Gamma, t \in(0, T)
\end{aligned}
$$

minimizing the performance functional

$$
I(v)=\lambda_{1} \int_{\Omega}\left|y(x, T ; v)-z_{d}\right|^{2} d x+\lambda_{2} \int_{\Omega}(N v) v d x
$$

where $\lambda_{i} \geq 0, \lambda_{1}+\lambda_{2}>0, z_{d}$ is a given element $L_{2}(\Omega)$ and $N$ is a positive linear operator.

Subaşı and Saraç [8] studied the problem of the determining the initial velocity of the linear hyperbolic problem by minimizing the cost functional

$$
J_{\alpha}(v)=\|u(x, T ; v)-y(x)\|_{L_{2}(0, l)}^{2}+\alpha\|v\|_{L_{2}(0, l)}^{2} .
$$

Lions [9] considered the following problem of minimizing the cost functional

$$
J(v)=\left\|y(T ; v)-z_{d}^{0}\right\|_{H_{0}^{1}(\Omega)}^{2}+\left\|y^{\prime}(T ; v)-z_{d}^{1}\right\|_{L_{2}(\Omega)}^{2}
$$

under the following condition

$$
\begin{aligned}
& y^{\prime \prime}(v)+A(t) y(v)=f \\
& y(0 ; v)=0, y^{\prime}(0 ; v)=v
\end{aligned}
$$

where $f \in L_{2}\left(0, T ; L_{2}(\Omega)\right), v \in L_{2}(\Omega)$ and the targets $z_{d}^{0} \in H_{0}^{1}(\Omega)$ and $z_{d}^{1} \in L_{2}(\Omega)$.

We organize this paper as follows. In section 2, we present the weak solution of the hyperbolic problem considered and gives necessary conditions for the existence and uniqueness of the optimal solution. In section 3, we get the Frechet differential of the cost functional via the solution of the corresponding adjoint problem. In last section, we constitute a minimizing sequence is constituted then the limit of this sequence is the solution of the optimal control problem considered.

\section{Unicity of solutions of the optimal control problem}

In this section, we give the solvability of the optimal control problem(1)-(2). First we state the generalized solution of the hyperbolic problem (2) in view of [10]. 
The generalized (weak) solution of the problem (2) will be defined as the function $u \in H_{0}^{1}(\Omega)$, with $u(x, 0)=\varphi(x), x \in(0, l)$ which satisfies the following integral identity:

$$
\int_{0}^{T} \int_{0}^{l}\left(-u_{t} \eta_{t}+a^{2} u_{x} \eta_{x}\right) d x d t=\int_{0}^{T} \int_{0}^{l} f v \eta d x d t+\int_{0}^{l} v \eta(x, 0) d x
$$

for all $\eta \in H_{0}^{1}(\Omega)$ with $\eta(x, T)=0$.

We know from [10], that for every $\varphi \in H_{0}^{1}(0, l), v \in L_{2}(0, l)$ and $F \in L_{2}(\Omega)$, the problem (2) has a unique generalized solution and the following estimate is valid for this solution;

$$
\|u\|_{H_{0}^{1}(\Omega)}^{2} \leq c_{0}\left(\|\varphi\|_{H_{0}^{1}(0, l)}^{2}+\|v\|_{L_{2}(0, l)}^{2}+\|F\|_{L_{2}(\Omega)}^{2}\right)
$$

Let's give the increment $\Delta v$ to $v$ such that $v+\Delta v \in U_{a d}$ and show the solution of (2) corresponding $v+\Delta v$ by $u_{\Delta}=$ $u(x, t ; v+\Delta v)$. Then the function $\Delta u=u_{\Delta}-u$ will be the solution of the following difference problem:

$$
\begin{aligned}
& \Delta u_{t t}=a^{2} \Delta u_{x x} \\
& \Delta u(x, 0)=0, \Delta u_{t}(x, 0)=\Delta v(x) \\
& \Delta u_{x}(0, t)=0, \Delta u_{x}(l, t)=0
\end{aligned}
$$

Lemma 1. Let $\Delta u$ be the solution of the problem (6). Then the following estimate is valid:

$$
\|\Delta u(0, .)\|_{L_{2}(0, T)} \leq c_{1}\|\Delta v\|_{L_{2}(0, l)}
$$

where $c_{1}=\sqrt{\frac{l T}{a^{2}}}$.

Proof. We can proof this lemma in view of [5]. We multiply both sides of the hyperbolic equation (6) by $\Delta u_{t}$, then integrate it on $[0, l]$. After some transformations, we obtain

$$
\frac{1}{2} \frac{d}{d t}\left\{\int_{0}^{l}\left[\left(\Delta u_{t}\right)^{2}+a^{2}\left(\Delta u_{x}\right)^{2}\right] d x\right\}=a^{2}\left(\Delta u_{x} \Delta u_{t}\right)_{x=0}^{x=l}
$$

Using here the homogeneous Neumann boundary conditions of the system (6), we write

$$
\frac{1}{2} \frac{d}{d t}\left\{\int_{0}^{l}\left[\left(\Delta u_{t}\right)^{2}+a^{2}\left(\Delta u_{x}\right)^{2}\right] d x\right\}=0
$$

We integrate both sides on $[0, t], t \in[0, T]$

$$
\int_{0}^{l}\left[\left(\Delta u_{t}\right)^{2}+a^{2}\left(\Delta u_{x}\right)^{2}\right] d x=\int_{0}^{l}[\Delta v(x)]^{2} d x, \quad \forall t \in[0, T] .
$$

Integrating now both the sides on $[0, T]$, we have

$$
\left\|\Delta u_{x}\right\|_{L_{2}(\Omega)}^{2}=\int_{0}^{T} \int_{0}^{l}\left(\Delta u_{x}\right)^{2} d x d t \leq \frac{T}{a^{2}} \int_{0}^{l}[\Delta v(x)]^{2} d x .
$$

To obtain estimate (7) needs to use the inequality $\|\Delta u(0, .)\|_{L_{2}(0, T)}^{2} \leq l\left\|\Delta u_{x}\right\|_{L_{2}(\Omega)}^{2}$ on the left hand side of (8). This complete the proof. 
We can write the cost functional (1) in the following way;

$$
J_{\alpha}(v)=\int_{0}^{T}[u(0, t ; v)-u(0, t ; 0)+u(0, t ; 0)-y(t)]^{2} d t+\alpha \int_{0}^{l} v^{2} d x
$$

So we rewrite $J_{\alpha}(v)$ as

$$
J_{\alpha}(v)=\pi(v, v)-2 L v+b
$$

for

$$
\begin{aligned}
& \pi(v, v)=\int_{0}^{T}[u(0, t ; v)-u(0, t ; 0)]^{2} d t+\alpha \int_{0}^{l} v^{2} d x \\
& L v=\int_{0}^{T}[u(0, t ; v)-u(0, t ; 0)][y(t)-u(0, t ; 0)] d t
\end{aligned}
$$

and

$$
b=\int_{0}^{T}[y(t)-u(0, t ; 0)]^{2} d t
$$

Due to the linearity of the transform $v \rightarrow u[v]-u[0]$, it can easily be seen that the functional $\pi(v, v)$ is bilinear and symmetric. Further, we write the following;

$$
|\pi(v, v)| \geq \alpha\|v\|_{L_{2}(0, l)}^{2}
$$

and this implies the coercivity of $\pi(v, v)$. Since

$$
\pi(v, \eta)=\int_{0}^{T}[u(0, t ; v)-u(0, t ; 0)][u(0, t ; \eta)-u(0, t ; 0)] d t+\alpha \int_{0}^{l} v \eta d x
$$

applying Cauchy-Schwartz inequality and using (7), we get

$$
|\pi(v, \eta)| \leq c_{2}\|v\|_{L_{2}(0, l)}\|\eta\|_{L_{2}(0, l)}
$$

for $c_{2}=\max \left\{c_{1}^{2}, \alpha\right\}$. Then $\pi(v, \eta)$ is continuous.

The functional $L v$ is linear. We can easily write that

$$
L v \leq c_{3}\|v\|_{L_{2}(0, l)}
$$

using (7). Hence we see that the functional $L v$ is continuous.

Theorem 1. Let $\pi(v, v)$ be a continuous symmetric bilinear coercive form and Lv be a continuous linear form. Then there exists a unique element $v_{*} \in U_{\text {ad }}$ such that

$$
J_{\alpha}\left(v_{*}\right)=\operatorname{Inf}_{v \in U_{a d}} J_{\alpha}(v)
$$

Proof of this theorem can easily be obtained by showing the weak lower semi-continuity of $J_{\alpha}$ same as in [9].

\section{Frechet differentiability of the cost functional}

Let us introduce the Lagrangian $L(u, v, z)$ given by

$$
L(u, v, z)=\int_{0}^{T}[u(0, t ; v)-y(t)]^{2} d t+\alpha \int_{0}^{l} v^{2} d x+\int_{0}^{T} \int_{0}^{l}\left(u_{t t}-a^{2} u_{x x}-F(x, t)\right) z d x d t
$$


Using the $\delta L=0$ stationarity condition, we have the following adjoint problem:

$$
z_{t t}+a^{2} z_{x x}=0, \quad z(x, T)=0, z_{t}(x, T)=0, z_{x}(0, t)=\frac{2}{a^{2}}[u(0, t ; v)-y(t)], z_{x}(l, t)=0
$$

Now, we investigate the variation of the functional $J_{\alpha}(v)$. The difference functional $\Delta J_{\alpha}(v)=J_{\alpha}(v+\Delta v)-J_{\alpha}(v)$ is such as

$$
\Delta J_{\alpha}(v)=\int_{0}^{T}[2 u(0, t ; v)-2 y(t)+\Delta u(0, t)] \Delta u(0, t) d t+\alpha \int_{0}^{l}(2 v+\Delta v) \Delta v d x .
$$

Here, the term

$$
2 \int_{0}^{T}[u(0, t ; v)-y(t)] \Delta u(0, t) d t
$$

must be evaluated. Using the problems (6) and (17), we have

$$
2 \int_{0}^{T}[u(0, t ; v)-y(t)] \Delta u(0, t) d t=-\int_{0}^{l} z(x, 0) \Delta v d x .
$$

So the relation (18) can be written as

$$
\Delta J_{\alpha}(v)=\int_{0}^{l}\{-z(x, 0)+2 \alpha v\} \Delta v d x+\int_{0}^{T}[\Delta u(0, t)]^{2} d t+\alpha \int_{0}^{l}(\Delta v)^{2} d x .
$$

Using Lemma 1 in the (19), we can write the following equality:

$$
\Delta J_{\alpha}(v)=\langle-z(x, 0)+2 \alpha v, \Delta v\rangle_{L_{2}(0, l)}+o\left(\|\Delta v\|_{L_{2}(0, l)}^{2}\right)
$$

We get the gradient

$$
J_{\alpha}^{\prime}(v)=-z(x, 0)+2 \alpha v
$$

with the definition $J_{\alpha}(v+\Delta v)-J_{\alpha}(v)=\left\langle J_{\alpha}^{\prime}(v), \Delta v\right\rangle_{L_{2}(0, l)}+o\left(\|\Delta v\|_{L_{2}(0, l)}^{2}\right)$ of Frechet differential at $v \in U_{a d}$.

\section{Constituting minimizing sequence and its convergence}

In this section, we construct a minimizing sequence using the gradient method. If $v_{k}$ is known $(k \geq 0)$ then $v_{k+1}$ is computed by the following formula:

$$
v_{k+1}=v_{k}-\beta_{k} J_{\alpha}^{\prime}\left(v_{k}\right)
$$

where $v_{0} \in U_{a d}$ is a given initial iteration and $J_{\alpha}^{\prime}\left(v_{k}\right)$ is the Frechet derivation accompanying the element $v_{k}$.

We see that

$$
J_{\alpha}\left(v_{k+1}\right)-J_{\alpha}\left(v_{k}\right)=\beta_{k}\left[-\left\|J_{\alpha}^{\prime}\left(v_{k}\right)\right\|^{2}+\frac{o\left(\beta_{k}\right)}{\beta_{k}}\right]<0
$$

for sufficiently small $\beta_{k}>0$.

Computations of the $\beta_{k}$ can be carried out by one of the methods shown in [11].

One of the following can be taken as a stopping criterion to the iteration process;

$$
\left\|v_{k+1}-v_{k}\right\|<\varepsilon_{1},\left|J_{\alpha}\left(v_{k+1}\right)-J_{\alpha}\left(v_{k}\right)\right|<\varepsilon_{2},\left\|J_{\alpha}^{\prime}\left(v_{k}\right)\right\|<\varepsilon_{3} .
$$


Now, we show that for a minimizing sequence $\left\{v_{k}(x)\right\}$, the convergence of $J_{\alpha}\left(\left\{v_{k}\right\}\right) \rightarrow J_{\alpha}\left(v_{*}\right)$ implies $\left\|v_{k}-v_{*}\right\|_{L_{2}(0, l)} \rightarrow$ 0 for $k \rightarrow \infty$ while $\alpha>0$ using the strongly convexity of the cost functional.

Lemma 2. The cost functional (1) is strongly convex with the strong convexity constant $\alpha$ :

$$
J_{\alpha}\left(\beta v_{1}+(1-\beta) v_{2}\right) \leq \beta J_{\alpha}\left(v_{1}\right)+(1-\beta) J_{\alpha}\left(v_{2}\right)-\alpha \beta(1-\beta)\left\|v_{1}-v_{2}\right\|_{L_{2}(0, l)}^{2}
$$

Proof. Let's prove the functional $\alpha\|v\|_{L_{2}}^{2}$ is strongly convex. For all $v_{1}, v_{2} \in U_{\text {ad }}$ and $\beta \in[0,1]$, we write

$$
\begin{aligned}
\alpha\left\|\beta v_{1}+(1-\beta) v_{2}\right\|_{L_{2}}^{2} & =\alpha \int_{0}^{l}\left(\beta v_{1}+(1-\beta) v_{2}\right)^{2} d x \\
& =\alpha \int_{0}^{l}\left[\beta v_{1}^{2}+(1-\beta) v_{2}^{2}-\beta(1-\beta)\left(v_{1}-v_{2}\right)^{2}\right] d x \\
& =\beta \alpha\left\|v_{1}\right\|_{L_{2}}^{2}+(1-\beta) \alpha\left\|v_{2}\right\|_{L_{2}}^{2}-\alpha \beta(1-\beta)\left\|v_{1}-v_{2}\right\|_{L_{2}}^{2}
\end{aligned}
$$

Hence the functional $\alpha\|v\|_{L_{2}}^{2}$ is strongly convex with the strong convexity constant $\alpha$.

Now, we show that the functional $\pi(v, v)$ defined by (10) is the strongly convex. Using (23) and linearity of the transform $v \rightarrow u[v]-u[0]$, we obtain

$$
\begin{aligned}
\pi\left(\beta v_{1}+(1-\beta) v_{2}, \beta v_{1}+(1-\beta) v_{2}\right) & =\int_{0}^{T}\left[\beta\left(u\left(0, t ; v_{1}\right)-u(0, t ; 0)\right)+(1-\beta)\left(u\left(0, t ; v_{2}\right)-u(0, t ; 0)\right)\right]^{2} d x \\
& +\beta \alpha\left\|v_{1}\right\|_{L_{2}}^{2}+(1-\beta) \alpha\left\|v_{2}\right\|_{L_{2}}^{2}-\alpha \beta(1-\beta)\left\|v_{1}-v_{2}\right\|_{L_{2}}^{2}
\end{aligned}
$$

or

$$
\begin{aligned}
\pi\left(\beta v_{1}+(1-\beta) v_{2}, \beta v_{1}+(1-\beta) v_{2}\right) & =\beta^{2} \int_{0}^{T}\left(u\left(0, t ; v_{1}\right)-u(0, t ; 0)\right)^{2} d x+(1-\beta)^{2} \int_{0}^{T}\left(u\left(0, t ; v_{2}\right)-u(0, t ; 0)\right)^{2} d x \\
& +2 \beta(1-\beta) \int_{0}^{T}\left(u\left(0, t ; v_{1}\right)-u(0, t ; 0)\right)\left(u\left(0, t ; v_{2}\right)-u(0, t ; 0)\right) d x \\
& +\beta \alpha\left\|v_{1}\right\|_{L_{2}}^{2}+(1-\beta) \alpha\left\|v_{2}\right\|_{L_{2}}^{2}-\alpha \beta(1-\beta)\left\|v_{1}-v_{2}\right\|_{L_{2}}^{2}
\end{aligned}
$$

Applying the $\varepsilon$-Cauchy inequality to the third right-hand side integral and taking $\varepsilon=1$, we get

$$
\begin{aligned}
\pi\left(\beta v_{1}+(1-\beta) v_{2}, \beta v_{1}+(1-\beta) v_{2}\right) & \leq \beta \int_{0}^{T}\left(u\left(0, t ; v_{1}\right)-u(0, t ; 0)\right)^{2} d x+(1-\beta) \int_{0}^{T}\left(u\left(0, t ; v_{2}\right)-u(0, t ; 0)\right)^{2} d x \\
& +\beta \alpha\left\|v_{1}\right\|_{L_{2}}^{2}+(1-\beta) \alpha\left\|v_{2}\right\|_{L_{2}}^{2}-\alpha \beta(1-\beta)\left\|v_{1}-v_{2}\right\|_{L_{2}}^{2}
\end{aligned}
$$

or

$$
\pi\left(\beta v_{1}+(1-\beta) v_{2}, \beta v_{1}+(1-\beta) v_{2}\right) \leq \beta \pi\left(v_{1}, v_{1}\right)+(1-\beta) \pi\left(v_{2}, v_{2}\right)-\alpha \beta(1-\beta)\left\|v_{1}-v_{2}\right\|_{L_{2}}^{2}
$$

Thus the functional $\pi(v, v)$ is strongly convex with the strong convexity constant $\alpha$.

Taking $v=\beta v_{1}+(1-\beta) v_{2}$ in $(9)$ and using the linearity of the functional $L v$, we obtain

$$
\begin{aligned}
J_{\alpha}\left(\beta v_{1}+(1-\beta) v_{2}\right) & \leq \beta \pi\left(v_{1}, v_{1}\right)+(1-\beta) \pi\left(v_{2}, v_{2}\right)-\alpha \beta(1-\beta)\left\|v_{1}-v_{2}\right\|_{L_{2}}^{2}-2\left(\beta L v_{1}+(1-\beta) L v_{2}\right)+b \\
& =\beta J_{\alpha}\left(v_{1}\right)+(1-\beta) J_{\alpha}\left(v_{2}\right)-\alpha \beta(1-\beta)\left\|v_{1}-v_{2}\right\|_{L_{2}}^{2}
\end{aligned}
$$


This complete the proof.

So, we can give the following theorem which states the convergence of the minimizer to optimal solution.

Theorem 2. Let $v_{*}$ be optimum solution of the problem (1)-(2). For the strongly convex functional $J_{\alpha}(v)$ with the convexity constant $\alpha$, the minimizing sequence satisfies the following inequality;

$$
\left\|v_{k}-v_{*}\right\|_{L_{2}(0, l)}^{2} \leq \frac{2}{\alpha}\left(J_{\alpha}\left(v_{k}\right)-J_{\alpha}\left(v_{*}\right)\right), \quad k=0,1,2, \ldots
$$

Proof. Proof of this theorem can be obtained in a similar way to [11]. We can write

$$
J_{\alpha}\left(\frac{1}{2} v_{k}+\frac{1}{2} v_{*}\right) \leq \frac{1}{2} J_{\alpha}\left(v_{k}\right)+\frac{1}{2} J_{\alpha}\left(v_{*}\right)-\alpha \frac{1}{4}\left\|v_{k}-v_{*}\right\|_{L_{2}(0, l)}^{2}
$$

by taking $\beta=\frac{1}{2}$ in the definition of the strongly convex functional.

From

$$
J_{\alpha}\left(v_{*}\right) \leq J_{\alpha}\left(\frac{1}{2} v_{k}+\frac{1}{2} v_{*}\right)
$$

we get

$$
J_{\alpha}\left(v_{*}\right) \leq \frac{1}{2} J_{\alpha}\left(v_{k}\right)+\frac{1}{2} J_{\alpha}\left(v_{*}\right)-\alpha \frac{1}{4}\left\|v_{k}-v_{*}\right\|_{L_{2}(0, l)}^{2}
$$

and then

$$
\left\|v_{k}-v_{*}\right\|_{L_{2}(0, l)}^{2} \leq \frac{2}{\alpha}\left(J_{\alpha}\left(v_{k}\right)-J_{\alpha}\left(v_{*}\right)\right)
$$

Hence the proof is done.

Remark. We can obtain the above results by taking $J_{\alpha}(v)=\int_{0}^{T}[u(l, t ; v)-y(t)]^{2} d t+\alpha \int_{0}^{l} v^{2} d x$ instead of the cost functional defined as (1) in the optimal control problem (1)-(2).

\section{Conclusion}

In the hyperbolic problem, the initial velocity can be controlled from the target $u(0, t ; v)$. Using minimizing sequence (20), which includes the solution of the adjoint problem, the optimal solution of the optimal control problem can be reached successfully.

\section{Competing interests}

The authors declare that they have no competing interests.

\section{Authors' contributions}

All authors have contributed to all parts of the article. All authors read and approved the final manuscript.

\section{References}

[1] Vasilyev, F.P., Numerical Methods for Solving Extremal Problems, Nauka, Moskow, 1988. 
[2] Kowalewski, A., Optimal Control of Distributed hyeperbolic systems with deviating arguments, In. J. Control, 73(11), 1026-1041, 2000.

[3] Lagnesea, J.E. and Leugeringb, G., Time-domain Decomposition of Optimal Control Problems for the Wave equation, System and Control Letters, 48, 229-242, 2003.

[4] Periago, F., Optimal shape and position of the support for the internal exact control of a string, Systems \& Control Letters, 58, 136-140, 2009.

[5] Hasanov, A., Simultaneous Determination of the Source Terms in a Linear Hyperbolic Problem from the Final Overdetermination: Weak Solution Approach, IMA J. Appl. Math., 74, pp. 1-19, 2009.

[6] Serovajsky, S., Optimal control for the Systems Described by Hyperbolic Equation with Strong Nonlinearity, Journal of Applied Analysis and Compuation, 3(2), 183-195, 2013.

[7] Kowalewski, A., Optimal Control via Initial State of an Infinite Order Time Delay Hyperbolic System, Proceedings of the $18^{\text {th }}$ International Conference on Process Control, 14-17 June, Tatranska Lomnica, Slovakia, 2011.

[8] Subaş1, M., and Saraç, Y., A Minimizer for Optimizing the Initial Velocity in a Wave Equation, Optimization, 61(3), 327-333, 2012.

[9] Lions, J. L., Optimal Control of Systems Governed by Partial Differential Equations, Springer-Verlag, New York, 1971.

[10] Ladyzhenskaya, O. A., Boundary Value Problems in Mathematical Physics, Springer, New York, 1985.

[11] İskenderov, AD, Tagiyev, RQ, Yagubov, QY, Optimization Methods, Çaşığlu, Bakü, 2002. 